\title{
Alleles of Insm 1 determine whether RIP1-Tag2 mice produce insulinomas or nonfunctioning pancreatic neuroendocrine tumors
}

\author{
Shinta Kobayashi ${ }^{1}$, Tanupriya Contractor ${ }^{2}$, Evan Vosburgh $\mathbb{1}^{2}$, Yi-Chieh Nancy Du ${ }^{3}$, Laura H. Tang ${ }^{4}$,
} Richard Clausen ${ }^{2}$ and Chris R. Harris $2,5,6$

\begin{abstract}
The two most common types of pancreatic neuroendocrine tumors (PanNETs) are insulinomas and nonfunctioning PanNETs (NF-PanNETs). Insulinomas are small, rarely metastatic tumors that secrete high amounts of insulin, and nonfunctioning PanNETs are larger tumors that are frequently metastatic but that do not secrete hormones. Insulinomas are modeled by the highly studied RIP1-Tag2 (RT2) transgenic mice when bred into a C57BI/6 (B6) genetic background (also known as RT2 B6 mice). But there has been a need for an animal model of nonfunctioning PanNETs, which in the clinic are a more common and severe disease. Here we show that when bred into a hybrid AB6F1 genetic background, RT2 mice make nonfunctioning PanNETs. Compared to insulinomas produced by RT2 B6 mice, the tumors produced by RT2 AB6F1 mice were larger and more metastatic, and the animals did not suffer from hypoglycemia or hyperinsulinemia. Genetic crosses revealed that a locus in mouse chromosome 2qG1 was linked to liver metastasis and to lack of insulin production. This locus was tightly linked to the gene encoding Insm 1, a beta cell transcription factor that was highly expressed in human insulinomas but unexpressed in other types of PanNETs due to promoter hypermethylation. Insm1-deficient human cell lines expressed stem cell markers, were more invasive in vitro, and metastasized at higher rates in vivo when compared to isogenic Insm1-expressing cell lines. These data demonstrate that expression of Insm 1 can determine whether a PanNET is a localized insulinoma or a metastatic nonfunctioning tumor.
\end{abstract}

\section{Introduction}

Neuroendocrine cells function by secreting hormones in response to neurological or metabolic stimuli. The insulin-producing beta cells of the pancreas are the bestknown example of neuroendocrine cells, because beta cell defects can result in diabetes. Neuroendocrine cells are also found in many other sites of the body, including the pituitary, thyroid, parathyroid, small and large intestine.

\footnotetext{
Correspondence: Chris R. Harris (harrisch@ca.rutgers.edu) ${ }^{1}$ Institute for Advanced Study, Princeton, NJ, USA

${ }^{2}$ Raymond and Beverly Sackler Foundation Laboratory, New Brunswick, NJ, USA Full list of author information is available at the end of the article.

These authors contributed equally: Shinta Kobayashi, Tanupriya Contractor
}

In order to maintain proper hormone balance, neuroendocrine cells are under tight growth regulation. However, neuroendocrine cells can become transformed and develop into neuroendocrine tumors. Transformation of pancreatic beta cells results in pancreatic neuroendocrine tumors (PanNETs). PanNETs are the second most common tumors of the pancreas, with an incidence of 1 per 200,000, and the incidence of PanNETs has been increasing rapidly ${ }^{1}$. PanNETs often metastasize to the liver.

For such an uncommon disease, PanNETs have been a surprisingly popular research subject for tumor biologists. This is partly due to the fact that PanNETs are produced 
by the RIP1-Tag2 tumor model (RT2), which was one of the very first transgenic mouse models for cancer ${ }^{2}$. PanNETs occur in RT2 mice due to expression of the SV40 $\mathrm{T}$-antigen oncoprotein (Tag) from a rat insulin promoter (RIP). Tumor formation in RT2 mice is rapid and synchronized, which facilitates the testing of both potential therapeutics and potential tumor genes. RT2 is also a rare example of a mouse model that has been validated pharmacologically. Sunitinib and rapamycin were shown to block growth of tumors in RT2 mice $e^{3,4}$; these drugs were subsequently tested in clinical trials ${ }^{5,6}$, and approved by the FDA for use in patients. Conversely, antibodies against IGF1 receptor failed to block tumor progression in RT2 mice, and subsequently failed in the $\operatorname{clinic}^{7,8}$. The clinical success of RT2 as a model organism also prompted a reexamination of the $\mathrm{Rb}$ pathway in human PanNETs, because $\mathrm{Rb}$ is inactivated by the SV40 Tantigen. This analysis led to discovery of Cdk4 and Cdk6 amplifications and high $\mathrm{Rb}$ phosphorylation in pancreatic neuroendocrine tumors, as well as the demonstration that PanNET cell lines responded to Cdk4/6 inhibition especially in combination with rapamycin ${ }^{9}$. This study helped lead to a clinical trial of a Cdk4/6 inhibitor in combination with rapamycin-analog everolimus (ClinicalTrials.gov identifier NCT03070301). In another trial, a combination of the VEGFR2 inhibitor sunitinib and the c-met inhibitor PF-04217903 blocked tumor progression in RT2 mice ${ }^{10}$; subsequently, a PanNET patient clinical trial was initiated to test the effects of cabozantinib, a single agent targeting both VEGFR2 and c-met ${ }^{11}$.

The liver metastasis found in patients with PanNETs can also be detected in RT2 mice, although the frequency of metastasis is generally low. Researchers have published many reports on genes that can increase the rate of metastasis in this mouse, including Igflr, Alk, Rhamm, Met, Bclx, and C5 (refs. ${ }^{10,12-16}$ ). Also, Csf1 has been shown to be a metastasis suppressor in RT2 mice ${ }^{17}$.

Clinically, metastasis correlates with whether or not PanNETs produce insulin. PanNETs producing insulin are called insulinomas and these tumors are rarely malignant or metastatic; conversely, non-insulinproducing PanNETs are often highly malignant and metastatic $^{18}$. Most of the non-insulin-producing PanNETs are "nonfunctioning" tumors (NF-PanNETs), sonamed because they do not overproduce any of the major pancreatic endocrine hormones ${ }^{18}$. NF-PanNETs are by far the most clinically important of the pancreatic neuroendocrine tumors. It has been estimated that about $85 \%$ of PanNETs are nonfunctioning, $10 \%$ are insulinomas, and the remaining tumors express other hormones such as gastrin or glucagon ${ }^{19}$. Patients with nonfunctioning PanNETs have a 5 -year survival rate of only $33 \%{ }^{19}$, whereas patients with insulinomas rarely die of their disease. Nonfunctioning tumors are also larger in size than insulinomas.

Here we demonstrate that the RT2 mouse model is capable of modeling both insulinomas and nonfunctioning PanNETs, with the specific disease dependent on the genetic background of the animals. In a hybrid AB6F1 genetic background, low expression levels of a beta cell transcription factor, Insm1, favors development of nonfunctioning tumors, whereas in a $\mathrm{C} 57 \mathrm{Bl} / 6$ genetic background, higher expression of Insm1 favors development of insulinomas. Amounts of Insm1 correlated with insulin production and with lack of metastasis, in both mice and patients.

\section{Results}

RT2 AB6F1 mice were generated by mating females from the inbred A/J genetic background to male RT2 B6 mice, which have an inbred $\mathrm{C} 57 \mathrm{Bl} / 6 \mathrm{~J}$ genetic background $^{12}$. For both genetic backgrounds, pancreatic tumors were produced that were neuroendocrine tumors upon pathological examination, albeit less welldifferentiated than the PanNETs found in most patients. There was a difference in frequency of liver metastasis in the two genetic backgrounds, with metastasis being much more common in RT2 AB6F1 mice than in RT2 B6 mice (Fig. 1a). Other genetic backgrounds have also been reported to influence metastasis frequency of RT2 mice ${ }^{13}$. Primary tumors were larger in age-matched RT2 AB6F1 mice compared to RT2 B6 (Fig. 1b). For RT2 AB6F1 animals, tumor size correlated with the presence of metastasis (Supplemental Fig. 1), while for RT2 B6 animals, a correlation between metastasis and tumor size was not tested because there were so few cases of animals with metastasis.

Remarkably, in spite of having larger, more metastatic tumors, RT2 AB6F1 mice actually lived longer than RT2 B6 mice (Fig. 1c). Eighty-five percentof RT2 AB6F1 mice but only 33\% of RT2 B6 mice lived to an age of 16 weeks. Early mortality may be related to hypoglycemia, which could be observed only in RT2 B6 mice and not in RT2 AB6F1 mice (Fig. 1d). Hypoglycemia has previously been reported in RT2 mice, so it was actually a surprise to find out that the RT2 AB6F1 were not hypoglycemic. In keeping with their hypoglycemia, RT2 B6 mice were also severely hyperinsulinemic, with an average nine-fold increase in serum insulin compared to wildtype B6 mice (Fig. 1e). Conversely, in spite of their larger pancreatic neuroendocrine tumors, RT2 AB6F1 expressed only twofold more serum insulin than wildtype AB6F1 mice. Immunohistochemistry revealed robust staining for insulin in tumors from RT2 B6 animals, but no insulin staining in tumors from RT2 AB6F1 animals (Supplemental Fig. 2). 
A

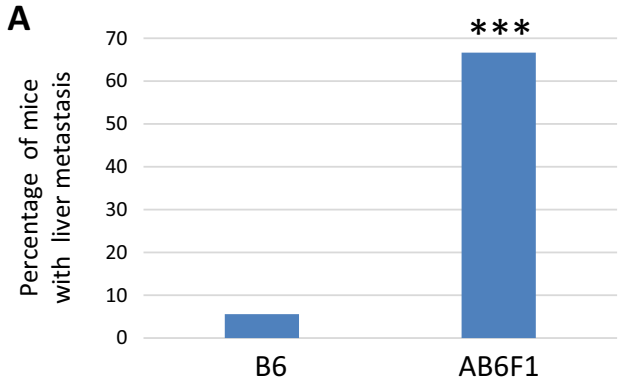

C

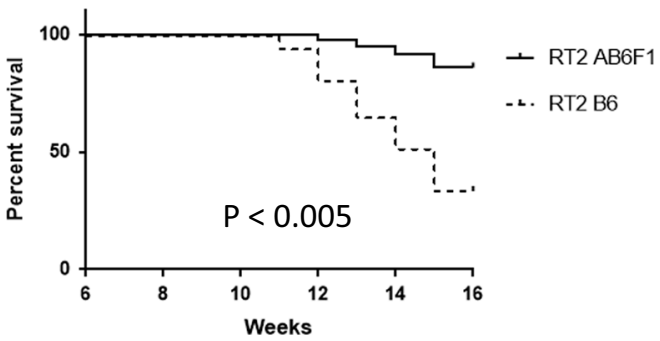

E

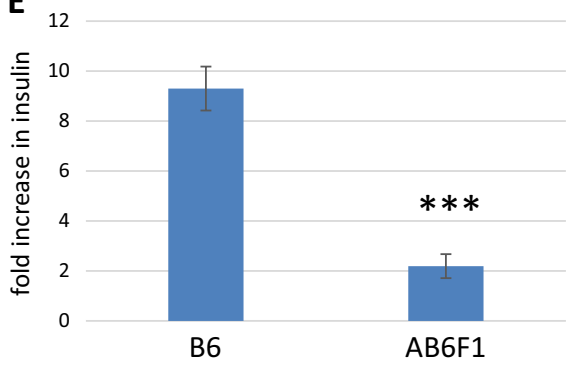

B

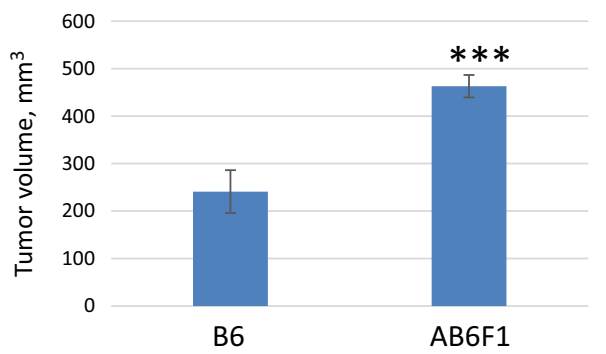

D

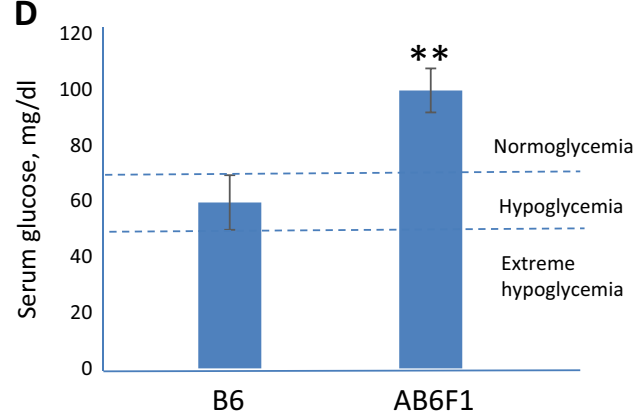

Fig. 1 RT2 B6 and RT2 AB6F1 mice show profound phenotypic differences. a Percentage of 15-week-old RT2 mice with liver metastasis, by genetic background. All mice were males, and 18 mice from each lineage were analyzed. Statistical significance was determined using Fisher's exact test. B6 mice have the C57BI/6 genetic background, and AB6F1 mice are hybrids resulting from mating female A/J mice to male C57BI/6 mice. $\mathbf{b}$ Volume of primary pancreatic tumors according to the genetic background. All mice were 17-week-old males; 24 RT2 B6 mice and 138 RT2 AB6F1 mice were analyzed. Statistical significance was determined using Mann-Whitney test. c Kaplan-Meier survival curves for RT2 mice between 6 and 16 weeks of age according to the genetic background. $\mathbf{d}$ Eight hours after removal of food, serum glucose was measured in RT2 mice of different genetic backgrounds. All mice were males and 12-13 weeks old. Fifteen RT2 AB6F1 and 12 RT2 B6 were analyzed. Statistical significance was determined using two-tailed t-test. e RT2 mice or wildtype littermates were held without food for $8 \mathrm{~h}$, then serum insulin was measured and compared to littermates (B6 or AB6F1) lacking the SV40 T-antigen transgene. Mice were males and 13 weeks old. Nine and 11 RT2 B6 and RT2 AB6F1 mice were measured, respectively. Statistical significance was determined using two-tailed t-test

Primary pancreatic tumors from both RT2 B6 and RT2 AB6F1 mice were harvested, and the latter expressed lower levels of mRNA for insulin (Fig. 2a), consistent with the low levels of serum insulin in these animals. Tumors from RT2 AB6F1 mice also transcribed less mRNA for other beta cell markers such as MafA, $P d x 1$, and $N k x 6-1$ (Fig. 2a). Interestingly, transcription of the gene for SV40 $\mathrm{T}$-antigen did not differ between tumors from RT2 AB6F1 and RT2 B6 mice (Fig. 2b), even though insulin expression differs strongly between the two mice. This may reflect the leakiness of the RIP that controls expression of SV40 $\mathrm{T}$-antigen; this transgene has also been shown to express in other insulin-negative neuroendocrine cells from small intestine and pituitary ${ }^{20,21}$.

With their smaller tumors, low metastasis, hypoglycemia, and hyperinsulinemia, RT2 B6 mice have all of the clinical features of human insulinomas. RT2 AB6F1 mice, on the other hand, may develop some other kind of pancreatic neuroendocrine tumor that does not express insulin. In RT2 AB6F1 tumors, transcription of glucagon and vasoactive intestinal peptide were not elevated, transcription of PPY and gastrin decreased, but transcription of somatostatin increased (Fig. 2c). Although elevated, the levels of somatostatin transcription were still 
A
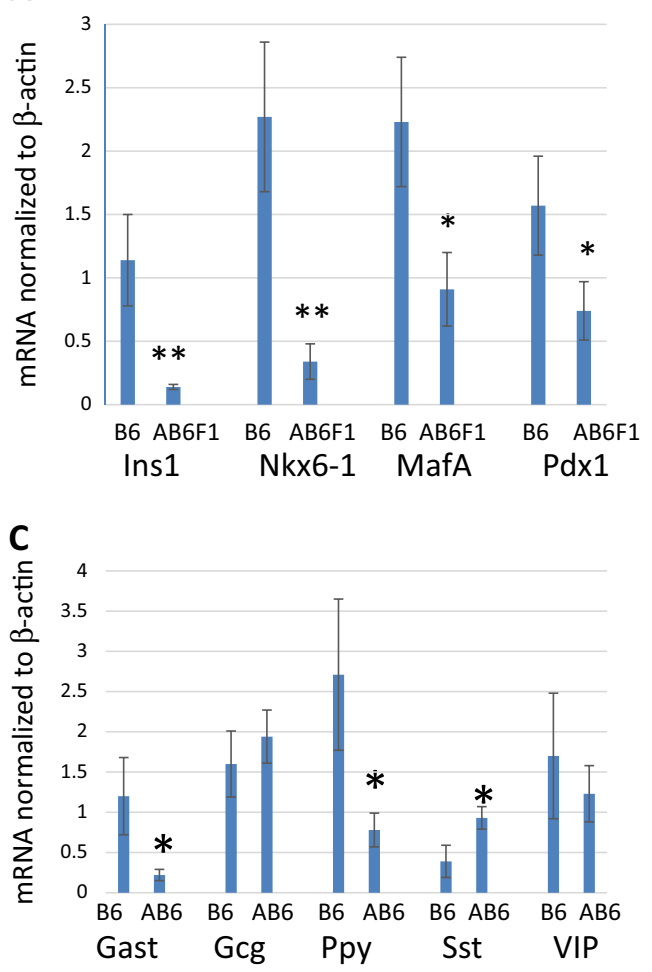

B

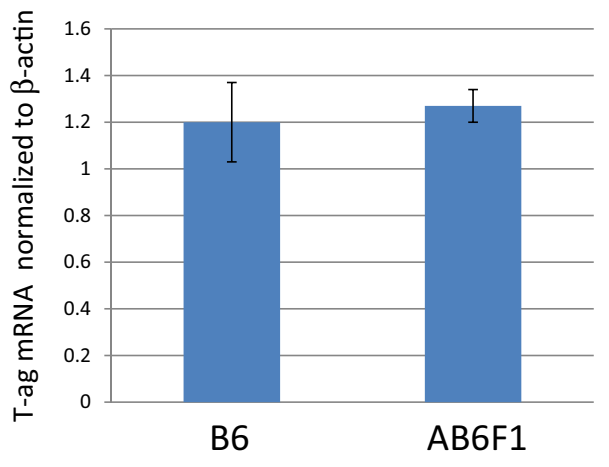

D

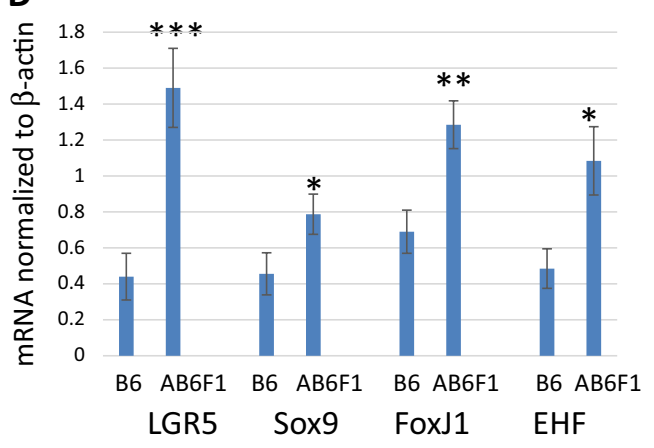

Fig. 2 Tumors from RT2 AB6F1 mice profile as nonfunctioning pancreatic neuroendocrine tumors. In each figure, Q-PCR was used to measure gene expression, using cDNA prepared from primary tumors isolated from 17 male RT2 B6 mice and from 25 male RT2 AB6F1 mice. All statistical analysis was performed using two-tailed t-test. a Beta cell markers insulin-1 (Ins 1), Nkx6-1, MafA, and Pdx1. b SV40 T-antigen, which is the oncogene that drives tumorigenesis in RT2 mice. c Pancreatic neuroendocrine hormones gastrin (Gast), glucagon (Gcg), pancreatic polypeptide (Ppy),

somatostatin (Sst), and vasoactive intestinal peptide (Vip). d Pancreatic stem cell markers Lgr5, Sox9, FoxJ1, and EHF

low. Patients with somatostatinomas suffer from weight loss and diarrhea, whereas RT2 AB6F1 were slightly overweight and had firm stools (data not shown). From these experiments, we conclude that tumors produced by RT2 AB6F1 mice profiled as nonfunctioning PanNETs, a clinically important disease in which patient tumors do not express high levels of any of the pancreatic hormones.

Thus in spite of sharing 50\% genetic identity, RT2 AB6F1 and RT2 B6 mice develop two very different types of PanNET. A possible clue for the genetic basis of this difference came from analysis of transcription of several markers of pancreatic stem cells ${ }^{22}$, which increased in tumors from RT2 AB6F1 mice (Fig. 2d). The loss of differentiation (Fig. 2a) but gain in stem cell markers (Fig. 2d) in RT2 AB6F1 tumors led to a hypothesis that $\mathrm{A} / \mathrm{J}$ and B6 mice may have different allelic forms of some beta cell differentiation factor.

Among the many genes known to be important for beta cell differentiation is Insm1, which can drive transdifferentiation of pancreatic ductal cells to endocrine cells ${ }^{23}$, presumably through a stem cell intermediate. Insm1knockout mice have also been reported to make beta cells that are deficient in expression of insulin ${ }^{24}$, which is perhaps similar to the deficient expression of insulin in tumors of RT2 AB6F1 mice. Notably, Insm1 was first isolated as a very highly expressed RNA in insulinomas ${ }^{25}$.

As shown in Fig. 3a, expression of Insm1 mRNA was higher in the insulinomas from RT2 B6 and lower in nonfunctioning tumors from RT2 AB6F1 mice. Insm1 protein levels were also higher in tumors from RT2 B6, as shown by western blotting (Fig. 3b). These experiments suggested that Insm1 might be relevant to the insulinomas and to the nonfunctioning tumors observed in RT2 mice.

Insm1 expression was characterized within a large set of patient samples. As shown in Fig. 3c, the mRNA of Insm1 strongly correlated with the mRNA of insulin. DNA was prepared from 16 of these human tumors, 8 of which expressed high amounts of insulin and 8 of which expressed very little insulin. Tumor DNAs were treated with bisulfite, and methylation-specific PCR was performed to test for DNA methylation within the promoter region of the Insm 1 gene. As shown in Fig. 3d, the Insm1 promoter was more strongly methylated in the tumors with low insulin and low Insm1. Since promoter 
A

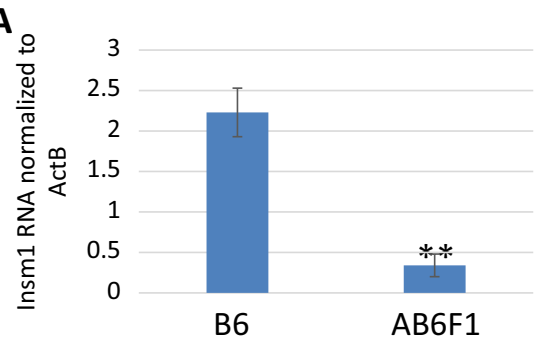

C

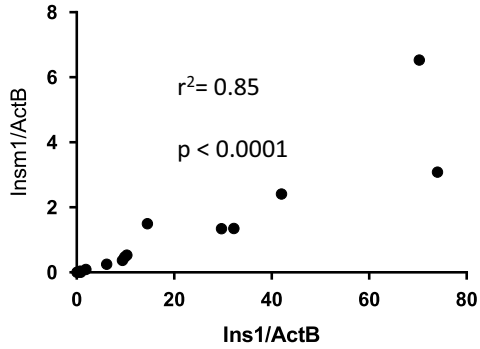

D

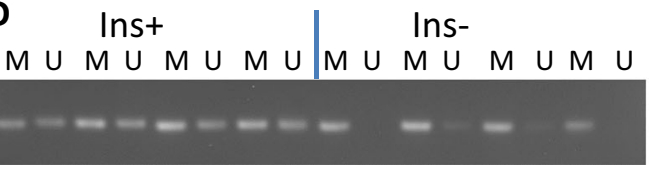

B

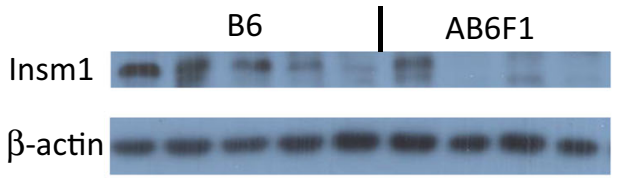

Fig. 3 Insulinomas from humans and mice express high levels of transcription factor Insm 1. a Q-PCR analysis of Insm 1 expression from primary tumors isolated from 17 male RT2 B6 mice or from 25 male RT2 AB6F1 mice. Statistical significance was determined using two-tailed t-test. $\mathbf{b}$ Western blot analyses of Insm 1 and $\beta$-actin expression from pancreatic neuroendocrine tumors of RT2 B6 or RT2 AB6F1 mice. c mRNA and cDNA were prepared from 39 human pancreatic neuroendocrine tumors, and transcription of beta cell markers Ins1 and Insm 1 was assayed by Q-PCR. Most of the tumors occupy the $(0,0)$ point of the graph. Statistical analysis was performed by Pearson correlation. d Tumor DNA from 16 patients was subjected to bisulfite treatment, followed by PCR using primers directed against a CpG island immediately upstream of the Insm 1 transcription start site. The results of PCR by Methylation-specific primer pairs are shown in lanes marked " $M$ ", and results of PCR by Unmethylation-specific primer pairs are shown in the lanes marked " $U$ ". In both gels, the first four tumors had high insulin expression by Q-PCR analysis, and the second four tumors had undectable insulin expression by Q-PCR analysis. Tumors were arbitrarily defined as having high insulin expression if they had an Ins $1 / A c t B$ mRNA ratio above 1.0 (see c). The eight insulin-negative tumors are likely to be nonfunctioning tumors, given the fact that $85 \%$ of PanNETs are nonfunctioning and $10 \%$ of PanNETs express insulin ${ }^{19}$. These eight insulin-negative tumors also had low expression of mRNA for gastrin and glucagon (data not shown)

hypermethylation is a common mechanism for lowering expression of key tumor suppressor genes, these data suggest that Insm1 expression may not merely correlate with insulinomas, but may actually be a gene that suppresses the formation of nonfunctioning PanNETs.

Next we tested whether allelic differences in the Insm1 genes encoded by the B6 and A/J lineages might be responsible for the insulinomas and NF-PNETs observed in RT2 B6 and RT2 AB6F1 mice, respectively. Insm1 genotypes were tested in a set of 279 RT2 AB6F2 mice, which had been prepared for a previous study ${ }^{12}$. RT2 AB6F2 are the product of mating RT2 AB6F1 mice. Unlike their hybrid F1 parents, which are genetically identical, F2 mice have a 1:2:1 Mendelian ratio of genotypes at all loci: one quarter of offspring will be homozygous for a given gene encoded by the A/J lineage, one quarter will be homozygous for the same gene encoded by the B6 lineage, and one half will be heterozygous at the locus. If a particular gene from the A/J genetic background promotes metastasis, then homozygotes and possibly heterozygotes for the $\mathrm{A} / \mathrm{J}$ allele of that gene should be more likely to show liver metastasis. A known SNP difference between the Insm1 alleles from C57Bl/6 and A/J mice was assayed.

As shown in Fig. 4a, the Insm1 SNP from the A/J background linked to metastasis in RT2 AB6F2 mice. Particularly notable is the fact that there was more metastasis in heterozygous mice compared to mice homozygous for the $\mathrm{B} 6$ allele of Insm1; this result matches with the fact that metastasis is more common in RT2 AB6F1 mice, which are heterozygous, than in RT2 B6 mice, which are homozygous for the B6 allele.

Importantly, if the differences in Insm1 alleles are responsible for the different types of PanNETs, then the Insm1 allele that links to metastasis should also link to lower insulin production. This is indeed the case. As 

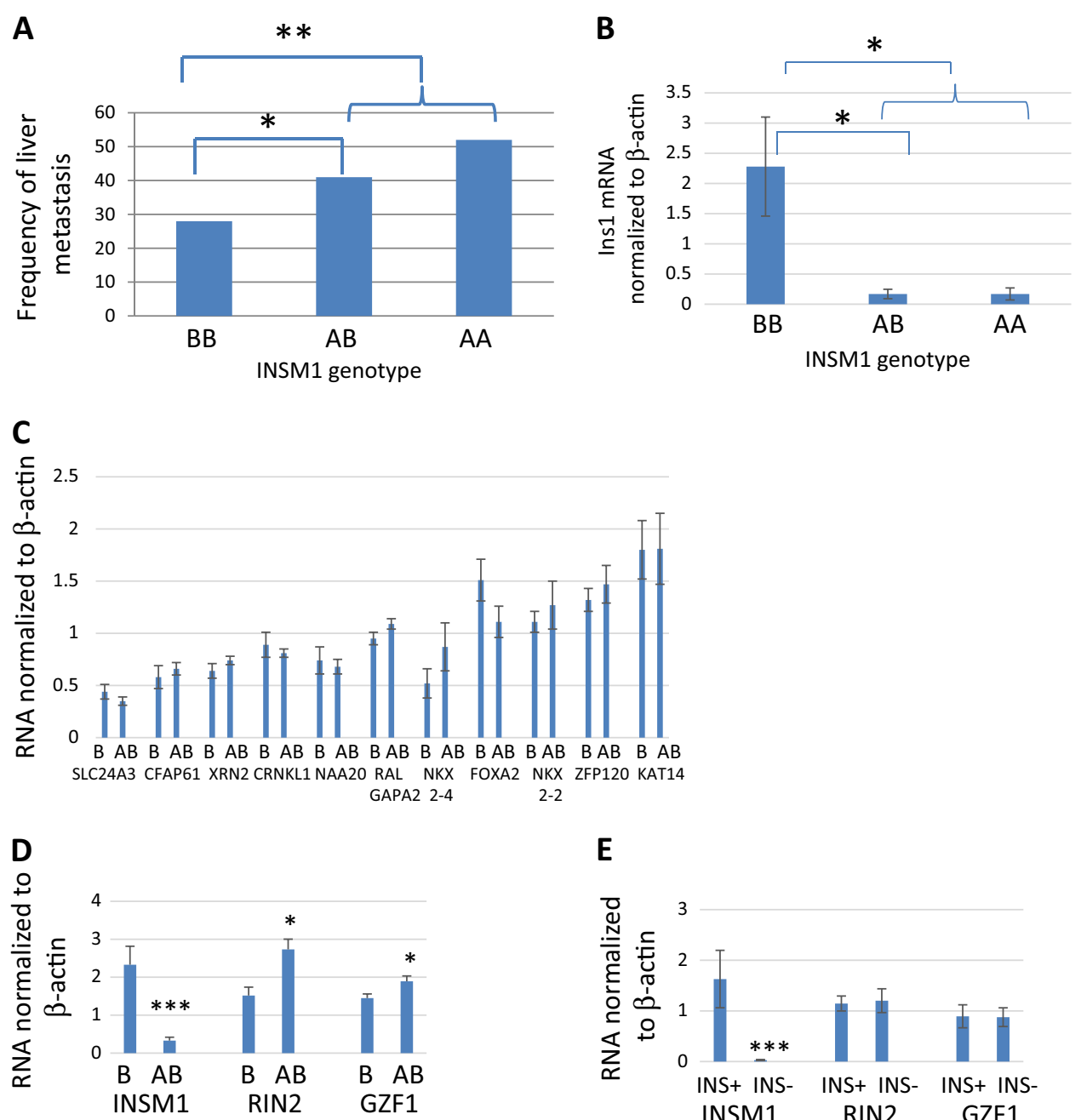

Fig. 4 The Insm 1 locus links to key phenotypic differences between RT2 B6 and RT2 AB6F1 mice. a RT2 AB6F2 mice were genotyped for a nucleotide difference within the Insm1 gene. Animals with two copies of the allele from the A/J lineage are called "AA", animals with two copies of the allele from the B6 lineage are called "BB," and heterozygous animals are called "AB". The mice were also scored for the presence of liver metastasis. A total of 279 mice were assayed. Statistical significance was computed using Fisher's exact test. b Transcription of insulin-1 (Ins 1 ) by 37 primary PanNETs from RT2 AB6F2 mice was compared using Q-PCR. The tumors were also genotyped to determine the Insm 1 genotype. Tumors with two Insm1 alleles from the B6 lineage are denoted "BB", tumors from heterozygous mice are denoted "AB," and tumors with two Insm1 alleles from the $A / J$ lineage are denoted as "AA". Statistical significance was determined using two-tailed $t$-test. c Q-PCR was used to analyze transcription of genes linked to Insm 1 in primary PanNETs from 18 RT2 B6 males and from 24 RT2 AB6F1 males. The total size of the region was 8 MeB, with Insm 1 in the center. All genes within $2 \mathrm{MeB}$ on either side of Insml were assayed, as well as a select number of candidate genes within 4 MeB on either side of Insm1. Candidate genes were selected as genes that were known to encode transcription factors and differentiation factors. "B" refers to tumors from RT2 B6 mice and "AB" refers to tumors from RT2 AB6F1 mice. mRNA for several genes within this region, including OvoL2, Kiz, and Pax1, could not be detected in spite of using two separate assays for each gene. $\mathbf{d}$ Transcription of Insm1, Rin2, and GZF1 in primary tumors from 18 RT2 B6 males and from 24 RT2 AB6F1 males was determined using Q-PCR. e Transcription of Insm1, Rin2, and GZF1 was determined for 39 primary patient pancreatic NETs. Tumors were arbitrarily defined as expressing insulin or not expressing insulin based on Ins1/ActB mRNA ratios above or below 1.0, respectively (see Fig. 3c)

shown in Fig. 4b, tumors from heterozygous animals expressed lower levels of mRNA for insulin than tumors from animals that inherited both of their Insm1 alleles from the B6 lineage. Again, this matches with the lower levels of insulin produced by RT2 AB6F1 mice, which are heterozygous, and with the higher levels of insulin produced by RT2 B6 mice, which are homozygous for the B6 allele. Thus, in F2 animals with at least one copy of Insm1 from the $\mathrm{A} / \mathrm{J}$ lineage, there was elevated metastasis and weak expression of insulin when compared to animals with two copies of Insm1 from the B6 lineage.

We also considered the possibility that a gene linked to Insm1, and not Insm1 itself, is the actual effector of tumor type in RT2 mice. If true, then perhaps such a gene would 
A

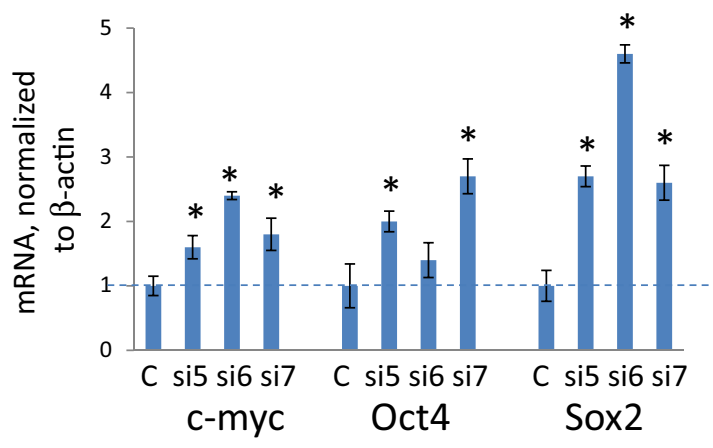

B

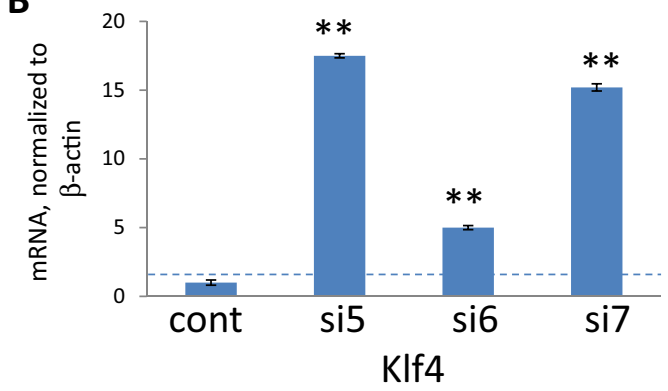

C

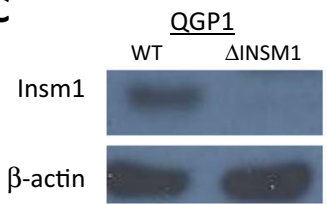

D
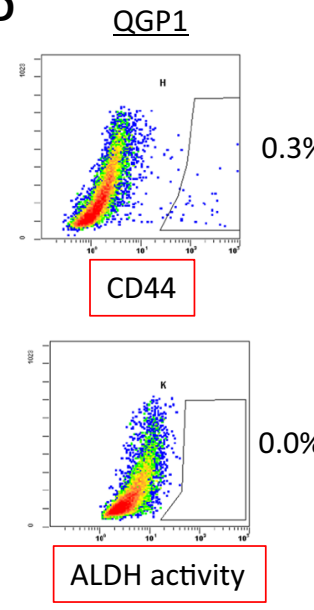

$\underline{\text { QGP1 } 1 \triangle I N S M 1}$
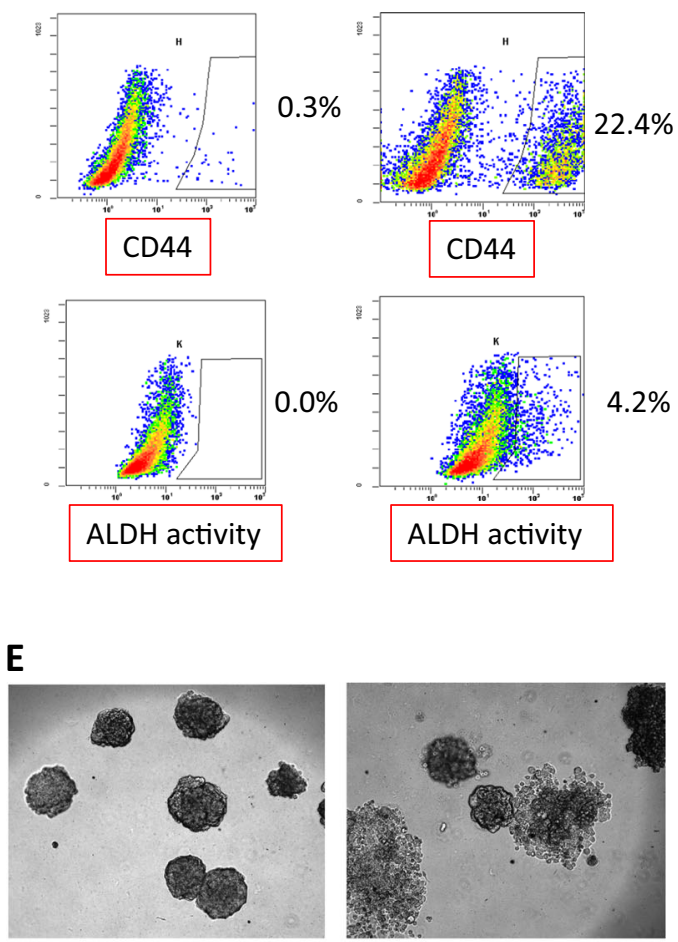

CD44-

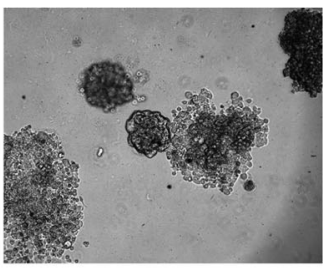

$\mathrm{CD}_{4} 4^{+}$

Fig. 5 Loss of Insm 1 expression promotes an invasive stem cell phenotype in human pancreatic neuroendocrine tumor cell line QGP1. a, b The QGP1 cell line was transfected with three different siRNAs directed against Insm1 (si-5, si-6, or si-7) or with control si-RNA (C, or cont). Eighty-four hours later, transcription of reprogramming genes Myc, Oct4, and Sox2 (a) or Klf4 (b) was measured by Q-PCR. c Western blot results, using an antibody against Insm1 to measure expression in the QGP1-TGL cell line, and in an isogenic version of QGP1-TGL in which the Insm 1 genes were knocked out using CRISPR-Cas9. Sequencing results for the knockout cell line are shown in the Materials and methods section. $\mathbf{d}$ The QGP1-TGL cell line expressing Insm1, as well as QGP1-TGL stably knocked out for the Insm1 gene, were tested for expression of stem cell markers CD44 and aldehyde dehydrogenase using flow cytometry. e Fluorescence activated cell sorting was used to collect CD44-positive and CD44-negative QGP-TGL cells, which were also knocked out for Insm1. The sorted cells were used to grow three-dimensional colonies in matrigel. CD44-negative cells formed sharply demarcated spheroids, but the colonies formed by CD44-expressing cells often lost their spheroid shape and appeared to invade the matrigel

show expression differences in RT2 B6 and RT2 AB6F1 tumors. Among genes residing within an $8 \mathrm{MeB}$ region of the mouse genome, centered at Insm1, three genes did show differences in expression between tumors from RT2 B6 and RT2 AB6F1 mice (Fig. 4c, d). However, of these three genes, only Insm 1 expression also showed differences in patient samples expressing high or low levels of insulin (Fig. 4e). Although this study does not completely rule out the possibility that another gene is involved, Insm1 does appear to be the best candidate.

If alleles of Insm 1 are indeed responsible for the two tumor subtypes, then Insm1 should be capable of blocking metastasis and dedifferentiation in neuroendocrine tumor cell lines. In Fig. 5a, b, the effect of transient knockdown of Insm1 expression using three different shRNAs was tested in the QGP1 cell line. Insm1 knockdown increased expression of the so-called Yamanaka factors ${ }^{26}$, which are known to increase pluripotency of differentiated cells. Next, CRISPR-Cas9 technology was used to stably knock out Insm1 from QGP1-TGL cells, a version of QGP1 engineered to express firefly luciferase (Fig. 5c). The Insm1 knockout increased the number of cells expressing aldehyde dehydrogenase and CD44, which are markers of stem cells (Fig. 5d). Insm1-null QGP1-TGL cells were then sorted for CD44 expression using flow cytometry. CD44-negative QGP1-TGL $\Delta$ Insm1 cells gave rise to spheroid colonies, whereas CD44-positive QGP1TGL $\Delta$ Insm1 cells produced colonies with invasive morphologies (Fig. 5e). These data indicate that loss of Insm1 can produce more invasive, stem-like cells. 

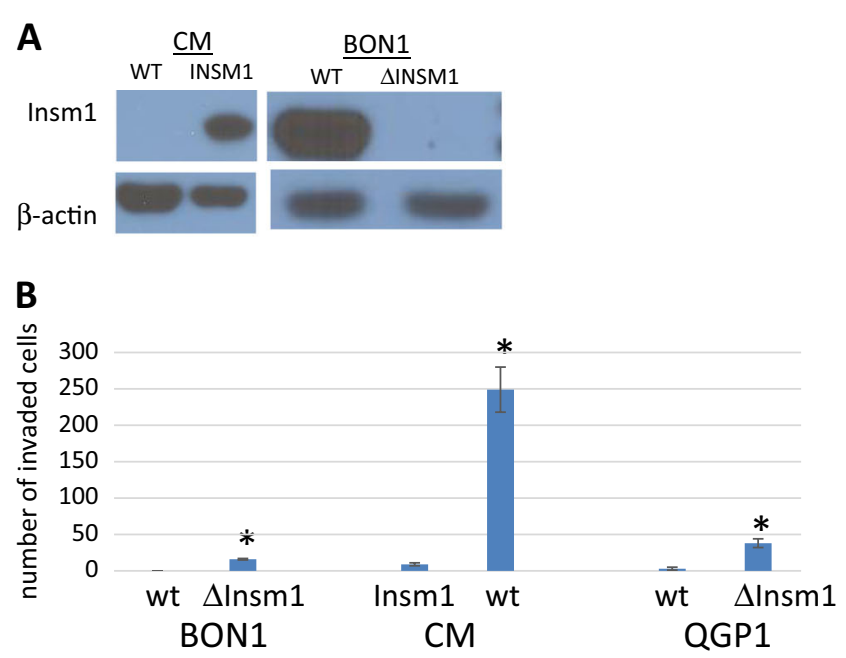

C

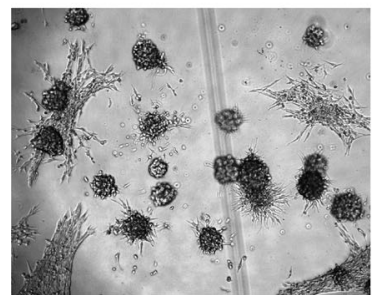

$\mathrm{CM}$ wt

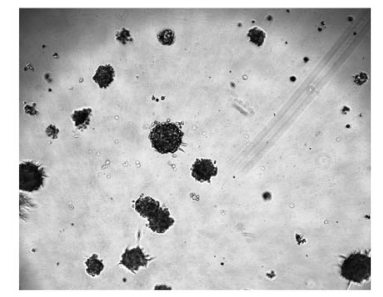

$\mathrm{CM}+\operatorname{lnsm} 1$

D

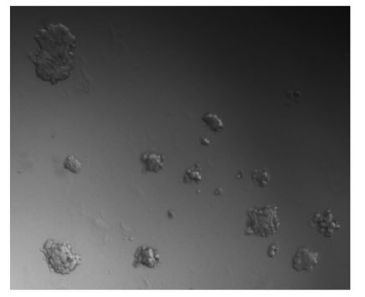

Bon1 $\Delta \operatorname{lnsm} 1$

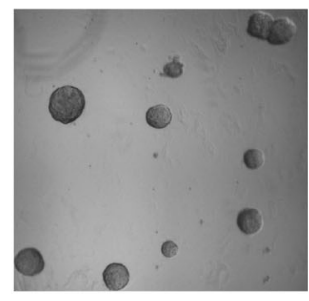

Bon1 wt

Fig. 6 Insm 1 expression decreases invasiveness of human pancreatic neuroendocrine tumor cell lines. a Western blot analysis, using antisera against Insm 1 or $\beta$-actin to analyze protein extracts from isogenic pairs of two cell lines (CM and BON1) in which Insm 1 was either expressed or not expressed. BON1 normally expresses Insm 1, so the two alleles were removed using CRISPR. CM cells do not normally express Insm I, so a plasmid expressing Insm 1 under control of the CMV promoter was introduced and a clone with physiological expression of Insm 1 was isolated. $\mathbf{b}$ Transwell assays were used to measure the ability of cells to migrate toward $20 \%$ serum across a membrane with $8 \mu \mathrm{m}$ pores, and then to invade matrigel. The tested cell lines were wildtype (wt) BON1, QGP1-TGL, or CM, along with isogenic versions of the cell lines that were engineered to alter their ability to express Insm1. c Single cells of the highly invasive CM cell line were grown into three-dimensional colonies for 2 weeks in matrigel. The presence of Insm1 changes the morphology of the colonies, decreasing their invasiveness and turning them spheroid. $\mathbf{d}$ Single cells of the BON1 cell line were grown into three-dimensional colonies for 2 weeks in matrigel. The cells deleted for Insm 1 have a less spheroid, more invasive morphology compared to wildtype cells, although this difference was not as dramatic as observed for CM in c

CRISPR-Cas9 was also used to knock out expression of Insm1 from a second Insm1-expressing PanNET cell line, BON1 (Fig. 6a). We also introduced Insm1 expression to the CM cell line, a highly undifferentiated PanNET cell line that did not normally express Insm1 protein (Fig. 6a). Along with QGP1-TGL, each of these isogenic pairs of Insm1-expressing and Insm1-null cell lines was tested for in vitro invasiveness, using a transwell assay. Invasiveness is a common property of metastatic cells. For all of the cell lines, invasiveness was higher if expression of Insm1 was absent, indicating that Insm1 is a repressor of invasion (Fig. 6b). The CM cell line was particularly invasive; the effects of Insm1 on this cell line could also be seen in three-dimensional culture, in which wildtype $\mathrm{CM}$ cells invade the surrounding matrix, but $\mathrm{CM}$ cells expressing Insm1 only form less invasive spheroids (Fig. 6c). Though less dramatic, Insm 1 also has an effect on the morphology of the BON1 cell line when grown in three-dimensional culture (Fig. 6d); the smaller change in morphology of BON1 correlated with the lower level of invasiveness of BON1 cells compared to CM cells.

Isogenic, Insm1-expressing, or Insm1-null QGP1-TGL cells were injected into the left ventricles of immune compromised mice. Mice were imaged weekly for bioluminescence of luciferase in order to assay for metastatic spread. Within 2 weeks, strong luciferase signals were detected in the torso, and these were particularly intense in animals injected with Insm1-null QGP1 (Fig. 7a). Luciferase signals also occurred above the neck; magnetic resonance imaging of the head revealed unusual growth of cells in the pre-nasal sinuses. Dissection of animals revealed that metastatic lesions were most prominent in the lung, liver, and pancreas. Examples are shown in Fig. $7 \mathrm{~b}$, where one of three animals injected with wildtype 

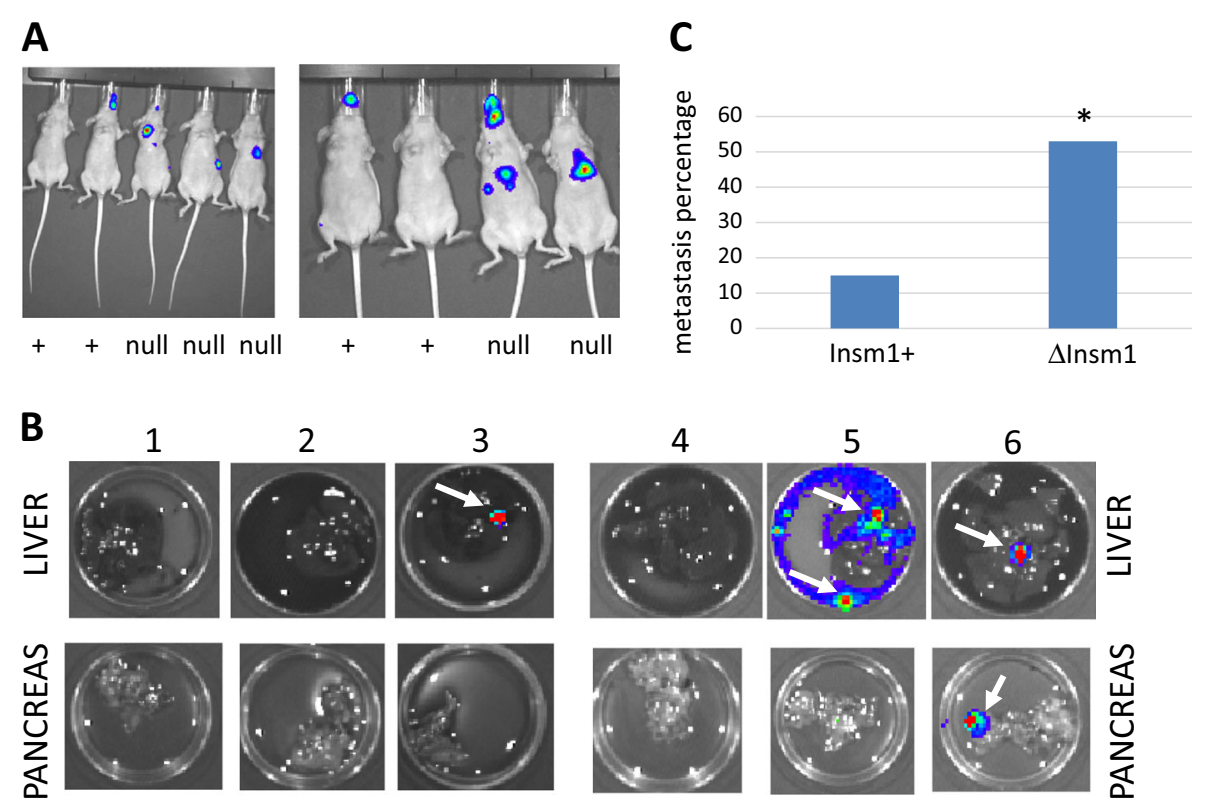

Insm1+

$\Delta \operatorname{lnsm1}$

Fig. 7 Insm 1 blocks in vivo metastasis by the QGP1-TGL cell line. a The QGP1 cell line was engineered to express firefly luciferase, and isogenic pairs either expressing Insm 1, or knocked out for Insm 1, were injected into the left ventricles of nude mice. Three weeks later, metastatic lesions were imaged in live mice following injection of luciferin. In both photos, the two mice on the left were injected with cells that express Insml ( + ), and the other animals were injected with cells unable to express Insm1 (null). b Three weeks after intracardial injection of luciferase-expressing QGP1 cells, mice were euthanized, and pancreas and liver were removed and treated with luciferin to help image metastases. The first three mice were injected with Insm1-expressing QGP1, and mice 4, 5, and 6 were injected with Insm 1-negative QGP1. Livers are in the upper row and pancreases are in the lower row. Metastases are noted by arrow. c Quantitation of metastases from lung, pancreas, and liver, which were isolated from 14 mice treated with luciferase-expressing QGP-TGL cells that either express or cannot express Insm 1. Metastasis was arbitrarily defined as individual organs giving radiance readings above $10^{5}$ photons $/ \mathrm{s} / \mathrm{cm}^{2} /$ steradian following luciferin treatment. Statistical significance was determined using Fisher's exact test

QGP-TGL produced a small lesion in the liver (animal number 3), but two of three animals (animals 5 and 6) injected with QGP-TGL knocked out for Insm1 produced lesions in the pancreas and in the liver. Altogether, the presence of metastatic tumors was 3.3-fold more common in the animals injected with the cell line knocked out for Insm1 (Fig. 7c).

Together, these cell line experiments establish that depletion of Insm 1 can cause stem-like cells to form, can increase invasiveness, and can cause metastasis; furthermore, these data suggest that the difference between the less metastatic, more differentiated insulinomas of RT2 B6 mice and the NF-PanNETs of RT2 AB6F1 mice may be due to differences in Insm1 expression. Coupled with the genetic linkage experiments from RT2 AB6F2 mice, the cell line data strongly suggest that allelic differences in Insm 1 between the $\mathrm{A} / \mathrm{J}$ and $\mathrm{B} 6$ mouse lines lead to different types of neuroendocrine tumors in RT2 mice.

\section{Discussion}

In two different genetic backgrounds (B6 and AB6F1), RT2 mice produced different types of PanNETs: insulinomas and nonfunctioning PanNETs, respectively. Several lines of evidence supported the idea that allelic differences in the Insm 1 gene were responsible for the difference in tumor types, and that Insm1 is a suppressor gene of nonfunctioning PanNETs. First and most importantly, in RT2 AB6F2 mice, the Insm1 locus linked strongly to high metastasis and to low insulin expression, which are two key characteristics of NF-PanNETs. Second, Insm1 expression was lower in NF-PanNETs, compared to insulinomas. Third, the Insm1 promoter was hypermethylated in human NF-PanNETs, as is often observed for tumor suppressor genes. Fourth, a key feature of NFPanNETs is metastasis, and knockout of Insm 1 was sufficient to increase in vivo metastasis of a pancreatic neuroendocrine cell line, QGP1-TGL1, and to increase in vitro invasiveness of additional neuroendocrine cell lines.

Insm 1 is a transcription factor that promotes beta cell differentiation ${ }^{27}$, and other tissue-specific differentiation factors have also been shown to suppress metastasis, such as GATA3 in breast tumors ${ }^{28}$ and NKX2-1 in lung tumors $^{29}$. It is generally thought that loss of these differentiation factors leads to metastasis by causing 
dedifferentiation to stem cells, and that stem cells increase metastasis because they are more invasive and less prone to anoikis ${ }^{30-32}$. Expression of stem cell markers increased in low Insm1-expressing tumors from RT2 AB6F1 mice (Fig. 2d). Upon knockout of Insm1 in the QGP1 cell line, stem cell markers increased, invasiveness increased, and metastasis increased.

The A/J and B6 lineages show several nucleotide differences within and near the Insm 1 locus, and it is possible that one or more of these differences affect Insm1 transcription or RNA stability. It is also formally possible that the Insm1 alleles in these lineages are not different at all and that another gene, linked to Insm1, can affect Insm1 expression. But we detected no evidence for such a mechanism, such as differential expression of another linked gene.

Interestingly, Insm 1 is not the only allelic difference between the $\mathrm{B} 6$ and $\mathrm{A} / \mathrm{J}$ lineages that affects neuroendocrine tumors. In our initial study of RT2 AB6F2 mice, we showed that the $\mathrm{A} / \mathrm{J}$ genome harbored a recessive, metastasis-suppressing locus, due to an inactivating mutation in the gene for $C 5$ (ref. ${ }^{12}$ ). But given that mating RT2 B6 with A/J mice produced an F1 generation with a very large increase in metastasis (Fig. 1a), we thought that the $\mathrm{A} / \mathrm{J}$ genome might also contain a metastasispromoting locus that was either dominant or co-dominant, and this allele turned out to be Insm1. Additionally, it has been shown that the Alk allele encoded by the B6 genome promotes metastasis more strongly than the Alk allele encoded by the $\mathrm{C} 3 \mathrm{H}$ inbred line ${ }^{13}$, and we also have evidence that the $\mathrm{B} 6$ allele of Alk promotes metastasis more strongly than the Alk allele of the A/J background (Contractor and Harris, unpublished data). Lastly, we have genetic data that metastasis in RT2 mice is also affected by yet another allelic difference between the $\mathrm{A} / \mathrm{J}$ and B6 lineages, but have not yet identified the actual gene (Contractor and Harris, unpublished data). Thus four, and possibly more, naturally occurring differences between the A/J and B6 mouse lines have a strong effect on whether the animal has a localized tumor or a metastatic one, an insulinoma or a NF-PanNET. Smallnucleotide polymorphisms that affect tumorigenesis have been identified for many tumor types ${ }^{33,34}$, but the high number of natural differences between $\mathrm{A} / \mathrm{J}$ and $\mathrm{B} 6$ that affect neuroendocrine tumorigenesis in RT2 mice makes one wonder whether NETs are peculiarly susceptible to naturally occurring genetic diversity.

The subtypes of pancreatic neuroendocrine tumors that can occur in patients show important clinical differences, with nonfunctioning PanNETs being more common and causing worse outcomes than insulinomas ${ }^{19}$. Understanding how different tumor subtypes can occur has been an active research area. In animal models for breast and brain tumors, it has been demonstrated that certain oncogene or tumor suppressor mutations can favor development toward one tumor subtype and not another ${ }^{35,36}$. In recently published work, mutations in Men1, Daxx, and Atrx were shown to be less common in human insulinomas than in human nonfunctioning tumors, suggesting that subtypes of PanNETs are influenced by the presence of these driver mutations ${ }^{37}$. But the present work also shows that tumor suppressor gene mutations are not sufficient to explain why different tumor subtypes can occur. Using mice that share a common driver oncogene, we show that tumor subtype is also influenced by naturally occurring genetic diversity, in the form of distinct alleles of the Insm1 gene. It would be interesting to know whether Insm1 expression can be directly influenced by mutations in Men1, Daxx, and Atrx, which encode chromatin-remodeling proteins. However, it should also be stated that mouse models do not always reflect how tumors develop in patients; indeed it is not clear whether low Insm1 expression is a direct cause of nonfunctioning PanNETs in patients, or a byproduct of the cell type from which these tumors originate ${ }^{38}$.

In patients, a key feature of NF-PanNETs is high rate of metastasis; NF-PanNETs in RT2 AB6F1 mice are likewise highly metastatic. The development of this mouse model may improve the ability to test drugs that could prevent metastasis of PanNETs in vivo. The RT2 mouse has previously been used for in metastasis studies, but comes with important shortcomings: metastasis is less penetrant in RT2 B6 mice, and is complicated by early death due to hypoglycemia. The RT2 AB6F1 mouse overcomes these issues. Metastasis is rapid and highly penetrant especially in male RT2 AB6F1 mice ${ }^{12}$, and RT2 AB6F1 mice are not hypoglycemic (Fig. 1d).

Several drugs have shown anti-metastatic activity in mouse models, but there have been no clinical trials designed to test drugs that might slow the risk of metastasis. This is in spite of the fact that metastasis is thought to be the cause of nearly all cancer deaths. There are several pragmatic reasons for the lack of trials on metastasis, including the fact that most clinical trials are performed on late stage, post-metastatic patients in order to overcome the high expense of lengthier trials on earlier stage patients. But another problem may be that spontaneous, synchronized mouse models of metastasis are rare. For this reason, the RT2 AB6F1 mouse model may be of great value. It derives from a mouse in which preclinical successes have led to human clinical trials and to FDA approvals. Although there is not a large population of patients with PanNETs, clinicians who study this disease are highly organized and have shown the ability to perform clinical trials that result in FDA approvals in spite of small patient sets. Patients with PanNETs are often detected before their tumors are metastatic, yet the onset of metastasis is fairly rapid for patients with 
nonfunctioning disease. All of these factors-a rapid and validated mouse model, a set of early diagnosed patients, fairly rapid onset of metastasis, and an organized set of clinicians-suggest that the RT2 AB6F1 mouse could prove to be an attractive preclinical model for developing and testing clinically relevant anti-metastatic agents.

\section{Materials and methods Cell lines}

The QGP1 cell line was purchased from the Japan Health Sciences Foundation. The BON1 cell line was a gift from the lab of Kjell Oberg and the CM cell line was a gift from the lab of Paolo Pozzilli. In order to authenticate the cell lines, short tandem repeat analysis was performed by ATCC. The STR results for QGP1 were a 100\% match to STR information for QGP1 found in the DSMZ database. The STR results for BON1 were a $100 \%$ match to previously published information ${ }^{39}$. STR results for CM did not match any other cell lines in the databases for ATCC, DSMZ, or EXPASY. STR results for the CM cell line have not previously been published, and are presented in Supplemental Fig. 3. QGP1 and BON1 have previously been tested for mycoplasma but CM cells were not. CM and QGP1 were grown in RPMI media (Thermofisher) supplemented with $10 \%$ fetal bovine serum (SigmaAldrich). BON1 cells were grown in Dulbecco's modified Eagle's medium (Thermofisher) with $10 \%$ fetal bovine serum. All lines were grown at $37^{\circ} \mathrm{C}$ under $5 \% \mathrm{CO}_{2}$. To decrease heterogeneity, the cell lines were cloned from single colonies before use. CM cells were engineered to express human Insm1 by using lipofectamine 2000 (Thermofisher) to transfect a plasmid expressing the human gene under control of the CMV promoter (Origene); Insm1-expressing cells were cloned from single transfectants. QGP1 and BON1 were engineered to express luciferase, and then Insm1 was knocked out by CRISPR, using an EDIT-R lentivirus purchased from Dharmacon (source clone identification number VSGHSM_26789438). Potential low expressers were cloned, and characterized by RTPCR and by western blotting, using an Insm1 antibody that was a generous gift from Mark Magnuson. Antisera against $\beta$-actin (part number A2228) were purchased from Sigma. Genomic DNA from low Insm1-expressing clones was subjected to PCR using primers $5^{\prime}$-CAGGTGTTCCCCTGCAAGTA and 5'-CCCAGACAACAGTTCAAGGC; the PCR products were cloned using the TOPO TA system (Thermofisher), and 12 of the PCR clones were sequenced to confirm the presence of frameshift mutations within the Insm1 sequence targeted by the EDIT-R lentivirus. BON1 clone 145 showed two new alleles of Insm1, each of which had frameshifting deletions: loss of the $10 \mathrm{nt}$ sequence ctcgcccggca, or loss of a single nucleotide, shown emboldened and in lower case within the following sequence: CCCGGCcTTACG. QGP1 clone H10 also showed two new alleles of Insm1, each of which had frameshifting mutations: loss of two nucleotides, CCCGGCctTACGCG, or loss of a single nucleotide, CCCGGCcTTACGCG. A matrigel-coated invasion chamber with $8 \mu \mathrm{m}$ pore size (Corning 354480 ) was used for invasion assays; $20 \%$ fetal bovine serum in the upper chamber was used as a chemo-attractant. Threedimensional growth of cell lines was performed by mixing 1000 cells with $50 \mu \mathrm{l}$ of matrigel (Corning 354234), and seeding $5 \mu \mathrm{l}$ of this mixture into 96 -well plates. After $5 \mathrm{~min}, 200 \mu \mathrm{l}$ of RPMI/10\% FBS media were added and the cells were cultured under $\mathrm{CO}_{2}$ at $37^{\circ} \mathrm{C}$ for 2 weeks. Insm 1 siRNAs si-5, si-6, and si-7 were purchased from Dharmacon, and catalog numbers were J-006535-05002, J-006535-06-002, and J-006535-07-002, respectively

\section{Human and mouse experiments}

Human pancreatic neuroendocrine tumors were provided by the Cooperative Human Tissue Network (CHTN), which obtained informed consents from patients. Human experiments were approved by the Institutional Review Board of CHTN. Mouse experiments were approved by the Institutional Animal Care and Use Committee of Rutgers University. RT2 B6 mice were obtained from the National Cancer Institute (Frederick, $\mathrm{MD}$ ) and bred to C57Bl6/J (Jackson laboratories) for more than 10 generations. A/J mice were purchased from Jackson Laboratories. Mouse husbandry, euthanasia, and autopsy protocols have been previously described ${ }^{12}$. Insm1 genotypes were not assayed until long after the mice had been euthanized and the metastasis and tumor size data were collected. To assay metastasis of the QGP1 cell line, one million cells expressing luciferase were injected into the left ventricles of nude mice, as previously described $^{14}$. The day after surgery, and weekly thereafter, mice were sedated with isofluorane, injected with luciferin, and imaged using an IVIS machine. Three weeks after injection, the mice were euthanized by $\mathrm{CO}_{2}$ asphyxiation and cervical dislocation, and autopsied for metastatic lesions. Organs were examined visually for metastatic lesions. Three organs (liver, pancreas, and both lungs) were also removed, treated with luciferin, and imaged using an IVIS machine. To assay serum insulin and serum glucose, mice were moved to fresh cages with water bottles but no food for $8 \mathrm{~h}$. An ELISA kit for mouse insulin was purchased from RayBiotech and used as recommended by the manufacturer. Serum glucose was measured using a ReliOn Confirm glucose meter and ReliOn Micro Plus test strips

\section{Flow cytometry analysis and cell sorting}

Cells were incubated with PE-labeled CD44 antibody (Biolegend) and 7-AAD Viability Dye (Beckman Coulter). 
Activity of aldehyde dehydrogenase was determined with AldeFluor Kit (Stem cell Technologies). Flow cytometry analysis and cell sorting were performed using a Cytomics FC500 Flow Cytometer (Beckman Coulter) or a BD Influx High Speed Cell Sorter (BD Biosciences). The purity of sorted cells was $>95 \%$.

\section{Genotyping}

An SNP assay directed against rs33272877, which differs between the Insm 1 alleles of $\mathrm{A} / \mathrm{J}$ and $\mathrm{C} 57 \mathrm{Bl} / 6$ genomes, was designed and synthesized by Thermofisher. Outside primer sequences were $5^{\prime}$-CGTGCTGGGCCTGAGT and $5^{\prime}$-GGGCGCCCTTGCT. Reporter sequences were 5'-AC CGCCCAGTGCCA and 5'-CCGCCGAGTGCCA. SNP analysis was perfomed using a Prism 7500 (Applied Biosystems).

\section{Analysis of RNA and protein}

Fresh-frozen tumor tissues from male mice were minced in Trizol (Thermofisher) and homogenized using a Polytron $1200 \mathrm{E}$. Chloroform was then added and the upper aqueous phase was removed after microcentrifugation at 14,000 r.p. $\mathrm{m}$. Ethanol was added and the RNA was then isolated using an RNAeasy column (Qiagen) as recommended by the manufacturer. RNA was converted to cDNA using reverse transcription reagents (Thermofisher). Real-time RTPCR was then performed, using a Prism 7500 (Applied Biosystems). Most of the RNAs were measured using pre-designed Taqman assays, which were purchased from Thermofisher. The following assays were designed with Primer3 (http:// bioinfo.ut.ee/primer3/) and run with SYBR green reagents (Thermofisher): mouse Slc24a3 (5'-CCCTCTGGCAAA CTGGAAAC and 5'-GGGATCCCTAGTGTGTAGCC); mouse Cfap61 (5'-AATCACTACCCTCAGCTGCA and 5'-GCCAAAGAAGCATGACCCAT); mouse Rin2 (5' -AACTCCTGGACCCATCATG and 5'-CATCCGCTGTT GACCTCTTG); mouse Xrn2 (5'-GAGGTCAAGCTCA GATCCCAAA and 5'-GTTCCATGGCAGTAGAGGTT CA); mouse Crnkl1 (5' AGAGAAGAAAGGTCCAGGCC and 5'-GCTTGAGGTTAGGCTGGTTG); mouse Naa20 (5'-AGGGAAGAATGGCATGGACA and 5'-GCTTGTA CATGTTGACGGCA); mouse Nkx2-4 (5'-GCCCCAT GAACCTGGAGATT and 5'-CACCTACCACATGCCTC CC); human Rin2 (5'-TCCGCACCATCTCCTGTTTC and 5'-GTCTGGACAAGCGAGGAAGT); mouse Sox 9 (5'TATCTTCAAGGCGCTGCAAG and 5'-CCCCTCTCGC TTCAGATCAA); mouse EHF (5'-GCCCGGCAGAAA GTCTTACT and 5'-TTCCAGTCCGCACACAATGT); mouse FoxJ1 (5'-CACTCTCATCTGCATGGCCA and 5' -AGGTTGTGGCGGATGGAATT); mouse Kat14 (5'-ACG AGAGGCTGAAACTGACA and 5'-ACGTCCACTTCCT TCCAGAG); mouse Zfp120 (5'-AAGCCCAGAAGTT CCGACAT and 5'-AGCAGCGAGATTCCTGTAGG); mouse GZF1 (5'-CTCAGCGCAATTCCCTGTAC and
5'-GTGAACTGCTTCCCACACTG); and human GZF1 (5'-TCACTCAGAACCACATGCTG and 5'-AATTCCGCT GGGCAAAAGTC.

\section{Analysis of promoter methylation}

Tumor DNA was treated using EpiMark Bisulfite Conversion Kit as recommended by the manufacturer (New England Biolabs). Primers were designed using MethPrimer 1.0 (ref. ${ }^{40}$ ). Methylation-specific primers were $5^{\prime}$-TTTATTTTACGCGGTTTATTTTTC and 5'ATCGAATCGAAATATTTATCTTCG. Unmethylationspecific primers were $5^{\prime}$-TGTTTGTTTTTATTTAATT AGTG and 5'-TCAAATCAAAATATTTATCTTCACC.

\section{Immunohistochemistry}

For antigen retrieval, slides were boiled for $16 \mathrm{~min}$ in $0.93 \%$ (v/v) Antigen Unmasking Solution H3301 (Vector Labs), and then slowly cooled to room temperature for $30 \mathrm{~min}$. Primary antibody was guinea pig anti-insulin from Dako (A0564), which was diluted 2000 -fold in $10 \%$ goat serum $/ 1 \%$ BSA. Slides were treated with primary antibody overnight at $4{ }^{\circ} \mathrm{C}$. Secondary antibody was affinity-purified, biotinylated antiguinea pig IgG from Vector Labs (BA-7000), which was diluted 500 -fold in $10 \%$ goat serum and $1 \%$ BSA. Slides were then treated with Vectastain Elite $\mathrm{ABC}$ peroxidase kit (Vector Labs) for $30 \mathrm{~min}$, and with ImmPact DAB peroxidase substrate (Vector Labs SK-4105) for 1min. The slides were counterstained with hematoxylin (Vector Labs).

\section{Statistical analysis}

Graphpad Prism 7.04 software was used for statistical analysis. Two-tailed $t$-test was used to compare RNA expression levels from sets of mouse and human tumors. Fisher's exact test was used to compare metastasis frequencies. Pearson correlation analysis was used to compare Insm 1 and Ins1 expression in human tumors. Nonparametric Mann-Whitney analysis was used to evaluate tumor volumes. Outliers were identified by Rout analysis $(q=1 \%)$.

\begin{abstract}
Acknowledgements
We thank the Raymond and Beverly Sackler Foundation, which funded this study. We also thank Arnie Levine and Robert Harris for very important insights, and Kjell Oberg, Mark Magnuson, and Paolo Pozzilli for contributing reagents. This research was supported by the Rutgers Molecular Imaging Center and by the Flow Cytometry/Cell Sorting shared resource of Rutgers Cancer Institute of New Jersey (P30CA072720).
\end{abstract}

\section{Author details}

${ }^{1}$ Institute for Advanced Study, Princeton, NJ, USA. ${ }^{2}$ Raymond and Beverly Sackler Foundation Laboratory, New Brunswick, NJ, USA. ${ }^{3}$ Weill Cornell

Medicine, New York, NY, USA. ${ }^{4}$ Memorial Sloan Kettering Cancer Center, New York, NY, USA. ${ }^{5}$ Rutgers University Cancer Institute of New Jersey, New Brunswick, NJ, USA. ${ }^{6}$ Department of Pediatrics, Robert Wood Johnson Medical School, New Brunswick, NJ, USA

Conflict of interest

The authors declare that they have no conflict of interest. 


\section{Publisher's note}

Springer Nature remains neutral with regard to jurisdictional claims in published maps and institutional affiliations.

Supplementary information accompanies this paper at (https://doi.org/ 10.1038/s41389-019-0127-1).

Received: 8 November 2018 Revised: 31 January 2019 Accepted: 8 February 2019

Published online: 22 February 2019

\section{References}

1. Kuo, E. J. \& Salem, R. R. Population-level analysis of pancreatic neuroendocrine tumors $2 \mathrm{~cm}$ or less in size. Ann. Surg. Oncol. 20, 2815-2821 (2013).

2. Hanahan, D. Heritable formation of pancreatic beta-cell tumours in transgenic mice expressing recombinant insulin/simian virus 40 oncogenes. Nature $\mathbf{3 1 5}$, 115-122 (1985).

3. Chiu, C. W., Nozawa, H. \& Hanahan, D. Survival benefit with proapoptotic molecular and pathologic responses from dual targeting of mammalian target of rapamycin and epidermal growth factor receptor in a preclinical model of pancreatic neuroendocrine carcinogenesis. J. Clin. Oncol. 28, 4425-4433 (2010).

4. Olson, P., Chu, G. C., Perry, S. R., Nolan-Stevaux, O. \& Hanahan, D. Imaging guided trials of the angiogenesis inhibitor sunitinib in mouse models predict efficacy in pancreatic neuroendocrine but not ductal carcinoma. Proc. Natl. Acad. Sci. USA 108, E1275-E1284 (2011).

5. Yao, J. C. et al. Everolimus for advanced pancreatic neuroendocrine tumors. $N$. Engl. J. Med. 364, 514-523 (2011).

6. Raymond, E. et al. Sunitinib malate for the treatment of pancreatic neuroendocrine tumors. N. Engl. J. Med. 364, 501-513 (2011).

7. Reidy-Lagunes, D. L. et al. A phase 2 study of the insulin-like growth factor-1 receptor inhibitor MK-0646 in patients with metastatic, well-differentiated neuroendocrine tumors. Cancer 118, 4795-4800 (2012).

8. Ulanet, D. B., Ludwig, D. L., Kahn, C. R. \& Hanahan, D. Insulin receptor functionally enhances multistage tumor progression and conveys intrinsic resistance to IGF-1R targeted therapy. Proc. Natl Acad. Sci. USA 107, 10791-10798 (2010).

9. Tang, L. H. et al. Attenuation of the retinoblastoma pathway in pancreatic neuroendocrine tumors due to increased cdk4/cdk6. Clin. Cancer Res. 18 4612-4620 (2012).

10. Sennino, B., Ishiguro-Oonuma, T., Schriver, B. J., Christensen, J. G. \& McDonald, D. M. Inhibition of c-Met reduces lymphatic metastasis in RIP-Tag2 transgenic mice. Cancer Res. 73, 3692-3703 (2013).

11. Chan, J. A. et al. Phase II trial of cabozantinib in patients with carcinoid and pancreatic neuroendocrine tumors (pNET). J. Clin. Oncol. 35(suppl), 228 (2017).

12. Contractor, T. et al. Sexual dimorphism of liver metastasis by murine pancreatic neuroendocrine tumors is affected by expression of complement C5. Oncotarget 7, 30585-30596 (2016).

13. Chun, M. G., Mao, J. H., Chiu, C. W., Balmain, A. \& Hanahan, D. Polymorphic genetic control of tumor invasion in a mouse model of pancreatic neuroendocrine carcinogenesis. Proc. Natl. Acad. Sci. USA 107, 17268-17273 (2010).

14. Choi, S. et al. BCl-xL promotes metastasis independent of its anti-apoptotic activity. Nat. Commun. 7, 10384 (2016).

15. Lopez, T. \& Hanahan, D. Elevated levels of IGF-1 receptor convey invasive and metastatic capability in a mouse model of pancreatic islet tumorigenesis. Cancer Cell. 1, 339-353 (2002).

16. Du, Y. C., Chou, C. K., Klimstra, D. S. \& Varmus, H. Receptor for hyaluronanmediated motility isoform $B$ promotes liver metastasis in a mouse model of multistep tumorigenesis and a tail vein assay for metastasis. Proc. Natl. Acad. Sci. USA 108, 16753-16758 (2011).
17. Pyonteck, S. M. et al. Deficiency of the macrophage growth factor CSF-1 disrupts pancreatic neuroendocrine tumor development. Oncogene 31, 1459-1467 (2012).

18. Anderson, C. W. \& Bennett, J. J. Clinical presentation and diagnosis of pancreatic neuroendocrine tumors. Surg. Oncol. Clin. N. Am. 25, 363-374 (2016).

19. Franko, J., Feng, W., Yip, L., Genovese, E. \& Moser, A. J. Non-functional neuroendocrine carcinoma of the pancreas: incidence, tumor biology, and outcomes in 2,158 patients. J. Gastrointest. Surg. 14, 541-548 (2010).

20. Biondi, C. A. et al. Conditional inactivation of the MEN1 gene leads to pancreatic and pituitary tumorigenesis but does not affect normal development of these tissues. Mol. Cell Biol. 24, 3125-3131 (2004).

21. Grant, S. G., Seidman, I., Hanahan, D. \& Bautch, V. L. Early invasiveness characterizes metastatic carcinoid tumors in transgenic mice. Cancer Res. 51, 4917-4923 (1991).

22. Dorrell, $\mathrm{C}$. et al. The organoid-initiating cells in mouse pancreas and liver are phenotypically and functionally similar. Stem Cell Res. 13, 275-283 (2014).

23. Zhang, T., Wang, H., Saunee, N. A., Breslin, M. B. \& Lan, M. S. Insulinomaassociated antigen-1 zinc-finger transcription factor promotes pancreatic duct cell trans-differentiation. Endocrinology 151, 2030-2039 (2010).

24. Gierl, M. S., Karoulias, N., Wende, H., Strehle, M. \& Birchmeier, C. The zinc-finger factor Insm 1 (IA-1) is essential for the development of pancreatic beta cells and intestinal endocrine cells. Genes Dev. 20, 2465-2478 (2006).

25. Goto, Y. et al. A novel human insulinoma-associated CDNA, IA-1, encodes a protein with "zinc-finger" DNA-binding motifs. J. Biol. Chem. 267, 15252-15257 (1992).

26. Takahashi, K. \& Yamanaka, S. Induction of pluripotent stem cells from mouse embryonic and adult fibroblast cultures by defined factors. Cell 126, 663-676 (2006).

27. Osipovich, A. B. et al. Insml promotes endocrine cell differentiation by modulating the expression of a network of genes that includes Neurog 3 and Ripply3. Development 141, 2939-2949 (2014).

28. Kouros-Mehr, H. et al. GATA-3 links tumor differentiation and dissemination in a luminal breast cancer model. Cancer Cell. 13, 141-152 (2008).

29. Winslow, M. M. et al. Suppression of lung adenocarcinoma progression by Nkx2-1. Nature 473, 101-104 (2011).

30. Lawson, D. A. et al. Single-cell analysis reveals a stem-cell program in human metastatic breast cancer cells. Nature 526, 131-135 (2015).

31. Mani, S. A. et al. The epithelial-mesenchymal transition generates cells with properties of stem cells. Cell 133, 704-715 (2008).

32. Hermann, P. C. et al. Distinct populations of cancer stem cells determine tumor growth and metastatic activity in human pancreatic cancer. Cell. Stem Cell. 1, 313-323 (2007).

33. Deng, N., Zhou, H., Fan, H. \& Yuan, Y. Single nucleotide polymorphisms and cancer susceptibility. Oncotarget 8, 110635-110649 (2017).

34. Post, S. M. et al. A high-frequency regulatory polymorphism in the p53 pathway accelerates tumor development. Cancer Cell. 18, 220-230 (2010).

35. Jacques, T. S. et al. Combinations of genetic mutations in the adult neural stem cell compartment determine brain tumour phenotypes. EMBO J. 29, 222-235 (2010).

36. Liu, J. C. et al. Combined deletion of Pten and p53 in mammary epithelium accelerates triple-negative breast cancer with dependency on eEF2K. EMBO Mol. Med. 6, 1542-1560 (2014).

37. Chan, C. S. et al. ATRX, DAXX or MEN1 mutant pancreatic neuroendocrine tumors are a distinct alpha-cell signature subgroup. Nat. Commun. 9, 4158 (2018).

38. Falchetti A. Genetics of multiple endocrine neoplasia type 1 syndrome: what's new and what's old. F1000Res. 6, F1000 Faculty Rev-73. https://doi.org/ 10.12688/f1000research.7230.1 (2017).

39. Hofving, T. et al. The neuroendocrine phenotype, genomic profile and therapeutic sensitivity of GEPNET cell lines. Endocr. Relat. Cancer 25, 367-380 (2018).

40. Li, L. C. \& Dahiya, R. MethPrimer: designing primers for methylation PCRs. Bioinformatics 18, 1427-1431 (2002). 